\title{
Updates to the HL7 2.5.1 Implementation Guide for Syndromic Surveillance
}

\author{
Peter Hicks ${ }^{1}$, Emilie Lamb ${ }^{2}$, Dave Trepanier ${ }^{2}$, Shandy Dearth ${ }^{2}$ \\ ${ }^{1} \mathrm{CDC}$, Atlanta, Georgia, United States \\ ${ }^{2}$ ISDS, Braintree, MA 02184, Massachusetts, United States
}

Objective

To describe the latest revisions and modifications to the "HL7 2.5.1 Implementation Guide for Syndromic Surveillance" (formerly the PHIN Message Guide for Syndromic Surveillance) that were made based on community commentary and resolution of feedback from the HL7 balloting process. In addition, the next steps and future activities as the IG becomes an "HL7 Standard for Trial Use" will be highlighted.

Introduction

In 2011, the Centers for Disease Control and Prevention (CDC) released the PHIN Messaging Guide for Syndromic Surveillance v. 1. In the intervening years, new technological advancements including Electronic Health Record capabilities, as well as new epidemiological and Meaningful Use requirements have led to the periodic updating and revision of the Message Guide. These updates occurred through informal and semi-structured solicitation and in response to comments from across public health, governmental, academic, and EHR vendor stakeholders. Following the Message Guide v.2.0 release in 2015, CDC initiated a multiyear endeavor to update the Message Guide in a more systematic manner and released further updates via an Erratum and a technical document developed with the National Institute of Standards and Technology (NIST) to clarify validation policies and certification parameters. This trio of documents were consolidated into the Message Guide v.2.1 release and used to inform the development of the NIST Syndromic Surveillance Test Suite (http://h17v2-ss-r2- testing.nist.gov/ss-r2/\#/home), validate test cases, and develop a new rules-based IG built using NIST's Implementation Guide Authoring and Management Tool (IGAMT).

As part of a Cooperative Agreement (CoAg) initiated in 2017, CDC partnered with ISDS to build upon prior activities and renew efforts in engaging the Syndromic Surveillance Community of Practice for comment on the Message Guide. The goal of this CoAg is have the final product become an "HL7 Standard for Trial Use" following the second phase of formal HL7 balloting $p$ in Fall 2018 .

\section{Methods}

ISDS coordinated a multi-stakeholder working group to revisit the consolidated Message Guide, v.2.1 and collect structured comments via an online portal, which facilitated the documentation, tracking, and prioritization of comments for developing consensus and reconciliation and resolution when there were errors, conflicts, or differing perspectives for select specifications. Over 220 comments were received during the most recent review period via the HL\& balloting process (April - June 2018) with sixteen elements captured for each comment, which included: Subject, Request Type, Clinical Venue Application, Submitter Name, IG Section \#, Priority, Working and Final Resolution (Figure 1). The online portal was used to communicate with members of the Message Guide Workgroup to provide feedback directly to one another through a 'conversation tab'. This became an important feature in teasing out underlying concerns and issues with a given comment across different local, state, and private sector partners (Figure 2). Some comments were able to be fully described and resolved using this feature. Following the HL7 balloting period, ISDS continued the weekly webinar-based review process to delve into specific issues in detail. Each week ISDS staff would lead the webinars structured around similar comment types (e.g. values sets, DG1 Segments, IN1 Segments, Conformance Statements, etc.). This leveraged the expertise of individuals and institutions with concerns revolving around a specific domain, messages segment, or specification described within the Message Guide. Comments for which consensus and resolution was achieved were "closed-out' on the portal inventory and new assignments for review would be disseminated across the Message Guide Workgroup for consideration and discussion during the subsequent webinar.

SDS Annual Conference Proceedings 2019. This is an Open Access article distributed under the terms of the Creative Commons AttributionNoncommercial 4.0 Unported License (http://creativecommons.org/licenses/by-nc/3.0/), permitting all non-commercial use, distribution, and reproduction in any medium, provided the original work is properly cited. 
ISDS 2019 Conference Abstracts

\section{Results}

To date this review process has identified and updated a wide-range of specification and requirements described within the Message Guide v.2.0. These include: specifications for persistent patient ID across venues of service, inclusion of the ICD-10-CM value set for diagnosis, removal of the ICD-9-CM requirement for testing and messages, modification of values such as pregnancy status, travel history, and medication list from "O" to "RE", and the update of value sets and PHIN VADS references for FIPS, SNOmed, ICD-10-CM, Acuity, Patient Class, and Discharge Disposition..

\section{Conclusions}

The results of this multi-agency comment and review process will be synthesized and compiled by ISDS. The updated version of the Message Guide (re-branded to the HL7 V 2.5.1 Implementation Guide for Syndromic Surveillance) will go through a second round of review and commentary thru HL7 in Fall 2018.

This systematic and structured review and documentation process has allowed for the synthetization and reconciliation of a wide range of disparate specifications, historical hold-overs, and requirements via the perspectives of a diverse range of public health partners. As this review process continues it is anticipated that the final HL7 balloted "Standard for Trial Use" IG 2.5 will represent a more refined and extensible product that can support syndromic surveillance activities across a wider and more diverse range of clinical venues, EHR implementations, and public health authorities.

ISDS and CDC have recommended that future modifications to the Promoting Interoperability (PI) Programs (formerly Meaningful Use) reference and require the utilization of the revised Implication Guide for Certification. The HL7 2.5.1 Implementation Guide can be found:

https://cdn.ymaws.com/www.healthsurveillance.org/resource/resmgr/docs/Group_Files/Message_Guide/IG_SyS_Release_1.pdf

\section{Acknowledgement}

We thank the surveillance professionals that assisted with the Implementation Guide. This work is supported Cooperative Agreement with the CDC. 